\title{
Walking in the Farey Fan to Compute the Characteristics of a Discrete Straight Line Subsegment
}

\author{
Isabelle Sivignon \\ gipsa-lab, CNRS, UMR 5216, F-38420, France \\ isabelle.sivignon@gipsa-lab.grenoble-inp.fr
}

\begin{abstract}
Given a Digital Straight Line (DSL) of known characteristics $(a, b, \mu)$, we address the problem of computing the characteristics of any of its subsegments. We propose a new algorithm as a smart walk in the so called Farey Fan. We take profit of the fact that the Farey Fan of order $n$ represents in a certain way all the digital segments of length $n$. The computation of the characteristics of a DSL subsegment is then equivalent to the localization of a point in the Farey Fan. Using fine arithmetical properties of the fan, we design a fast algorithm of theoretical complexity $\mathcal{O}(\log (n))$ where $n$ is the length of the subsegment. Experiments show that our algorithm is faster than the one previously proposed by Said and Lachaud in 1514 for "short" segments.
\end{abstract}

Keywords: Digital geometry, Digital straight segment recognition, Farey fan.

\section{Introduction}

Digital Straight Lines (DSL) and Digital Straight Segments (DSS) have been known for many years to be interesting tools for digital curve and shape analysis. The applications range from simple coding to complex multiresolution analysis and geometric estimators. All these applications require to solve the so-called DSS recognition problem. Many algorithms, using arithmetics, combinatorics or dual-space have been proposed to solve this problem, reaching a computational complexity of $\mathcal{O}(n)$ for a DSS of length $n$. A DSS belongs to infinitely many DSL of different characteristics, only one DSL enables to define the minimal characteristics of a DSS. In 14, the authors introduce the following problem: given a DSL of known characteristics and a subsegment of this DSL, compute the minimal characteristics of the DSS. The authors originally encountered this problem for implementing a fast algorithm to compute a multiresolution representation of a contour. This problem also arises for the digitization of a segment given by its two floating-point endpoints: indeed, the slope computed from the endpoints may be quite far from the minimal characteristics of the digitized segment, especially if the segment is short. Two algorithms (SmartDSS and ReversedSmartDSS) are presented in 1415]: both use the decomposition into continuous fractions of the DSL slope and reach a logarithmic complexity.

R. Gonzalez-Diaz, M.-J. Jimenez, B. Medrano (Eds.): DGCI 2013, LNCS 7749, pp. 23-34, 2013.

(C) Springer-Verlag Berlin Heidelberg 2013 
This problem is however not so new since in [12, the author presents a quick sketch of a method that solves it using the Farey Fan. The announced complexity of the method is $\mathcal{O}\left(\log ^{2} n\right)$ for a segment of length $n$. In this paper, we investigate further in this direction to provide a thoroughly defined algorithm. Moreover, we show how its complexity can be lowered to $\mathcal{O}(\log (n))$ with an astute use of arithmetical properties of the Farey Fan. Finally, we compare the performance of our algorithm with the ones proposed in 14 and 15 and show that it behaves particularly well for "short" segments.

\section{Setting the Problem}

\subsection{Digital line, Segment and Minimal Characteristics}

A Digital Straight Line (DSL for short) of integer characteristics $(a, b, \mu)$ is the infinite set of digital points $(x, y) \in \mathbb{Z}^{2}$ such that $0 \leq a x-b y+\mu<\max (|a|,|b|)$ $(\operatorname{gcd}(a, b)=1)[5]$. These DSL are 8-connected and often called naive. The slope of the DSL is the fraction $\frac{a}{b}$ and $\frac{\mu}{b}$ is the shift at the origin. In the following, without loss of generality, we assume that $0 \leq a \leq b$. The remainder of a DSL of characteristics $(a, b, \mu)$ for a given digital point $(x, y)$ is the value $a x-b y+\mu$. The upper (resp. lower) leaning line of a DSL is the straight line $a x-b y+\mu=0$ (resp. $a x-b y+\mu=b-1$ ). Upper (resp. lower) leaning points are the digital points of the DSL lying on the upper (resp. lower) leaning lines.

A Digital Straight Segment (DSS) is a finite 8-connected part of a DSL. It can be uniquely defined by the characteristics of a DSL containing it and two endpoints $P$ and $Q$. However, a DSS belongs to an infinite number of DSLs. In this context, the minimal characteristics of a DSS are the characteristics of the DSL containing it with minimal $b$ [16]. Note that the notions of leaning points and lines are similarly defined for DSSs. DSS recognition algorithms aim at computing the minimal characteristics of a DSS, taking profit of the following fact: $(a, b, \mu)$ are the minimal characteristics of a DSS if and only if the DSS contains at least three leaning points [5]. In this case, the minimal characteristics are the characteristics of the DSS upper leaning line.

The set of DSLs containing a DSS is usually called the preimage of the DSS. Given a DSS $S$, it is defined as $\mathcal{P}(S)=\{(\alpha, \beta),|\alpha| \leq 1 \mid \forall(x, y) \in S, 0 \leq$ $\alpha x-y+\beta<1\}$. The preimage can be represented in the $(\alpha, \beta)$ space, where $\alpha$ represents the slope and $\beta$ the shift at the origin of a straight line.

The preimage of a DSS is a polygon with a well-defined structure that is directly related to the leaning points and lines defined by its minimal characteristics 126]. Figure 1 below (from [4]) illustrates this point.

Proposition 1 ([4]). Let $\mathcal{P}(S)$ be the preimage of $S$. Let $A B C D$ be the polygon defined by this preimage, where $A$ is the upper left most vertex, and the vertices are named counterclockwise. Following the notations of Figure 1 we have:

- The vertex $B$ maps to the upper leaning line $U U^{\prime}$;

- The vertex $D$ maps to the lower leaning line $L L^{\prime}$ translated by the vector $(0,1)$ in the digital space; 


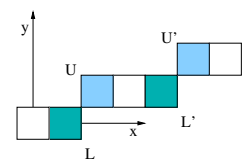

(a)

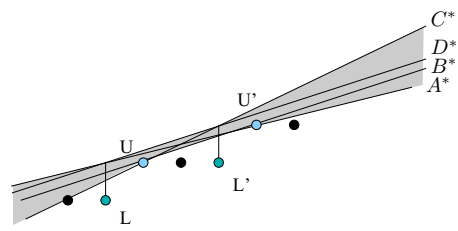

(b)

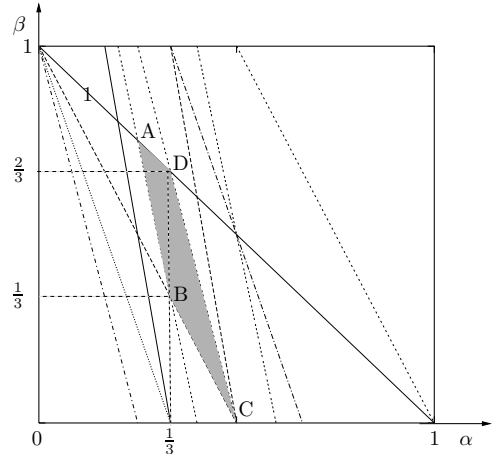

(c) (b)

Fig. 1. (a) DSS of minimal characteristics $(1,3,1)$ with its leaning points $U, U^{\prime}, L, L^{\prime}$. (c) Representation of $\mathcal{P}(S)$ is the $(\alpha, \beta)$ space.(b) Each vertex of the preimage maps to a straight line in the digital space. The vertex $B\left(\frac{1}{3}, \frac{1}{3}\right)$ maps to the upper leaning line, the characteristics of which are the minimal characteristics of the DSS.

- The vertex $A$ maps to the straight line $U^{\prime} L^{+}$, where $L^{+}=L+(0,1)$;

- The vertex $C$ maps to the straight line $U L^{\prime+}$, where $L^{\prime+}=L^{\prime}+(0,1)$.

The minimal characteristics of $S$ are $(a, b, \mu)$ if and only if $B=\left(\frac{a}{b}, \frac{\mu}{b}\right)$ ( $p$ and $q$ relatively prime). $B$ is called the characteristic point of $\mathcal{P}(S)$. Edges $[A B]$ and $[B C]$ are called lower edges.

\subsection{Farey Fan}

Definition 1 (Ray). Let $x$ and $y$ be two nonnegative integers. The ray defined by $x$ and $y$ is defined and denoted as follows:

$$
R(x, y)=\{(\alpha, \beta) \mid \beta=-x \alpha+y\}
$$

The slope of the ray is $x$.

Note that $x$ is not the geometrical slope of the ray but its absolute value. In the following, the order on the slopes is to be understood as the order on the absolute values of the geometrical slopes.

Definition 2 (Farey Fan). The Farey Fan of order $n$, denoted by $\mathcal{F}_{n}$ is defined in the $(\alpha, \beta)$ space as the arrangement of all the rays $R(x, y)$ such that $0 \leq y \leq$ $x \leq n$, and such that $0 \leq \alpha \leq 1$ and $0 \leq \beta \leq 1$.

A facet of $\mathcal{F}_{n}$ is a cell of dimension 2 of this arrangement. In the following, a point of $\mathcal{F}_{n}$ stands for any point $v$ of the $(\alpha, \beta)$ space $(0 \leq \alpha \leq 1$ and $0 \leq \beta \leq 1)$ belonging to a ray, and such that the abscissa of $v$ is a fraction of denominator smaller than or equal to $n$.

For any $n$, it is well known that there is a bijection between the facets of $\mathcal{F}_{n}$ and the set of DSSs of length $n$ (composed of $n+1$ pixels) [12]. 


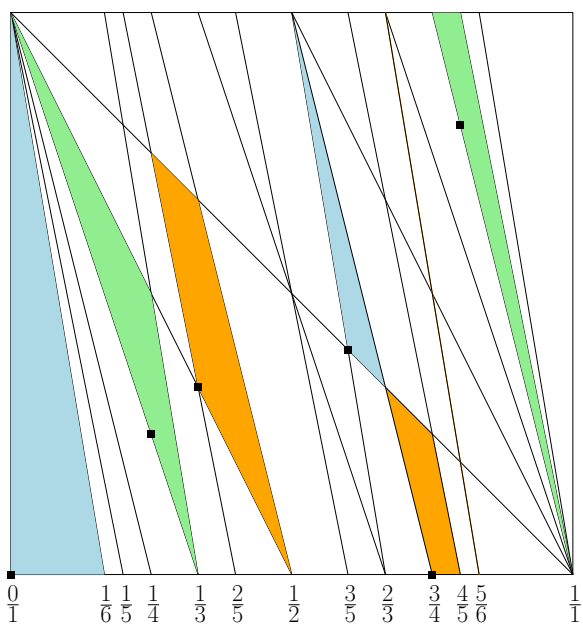

(a)

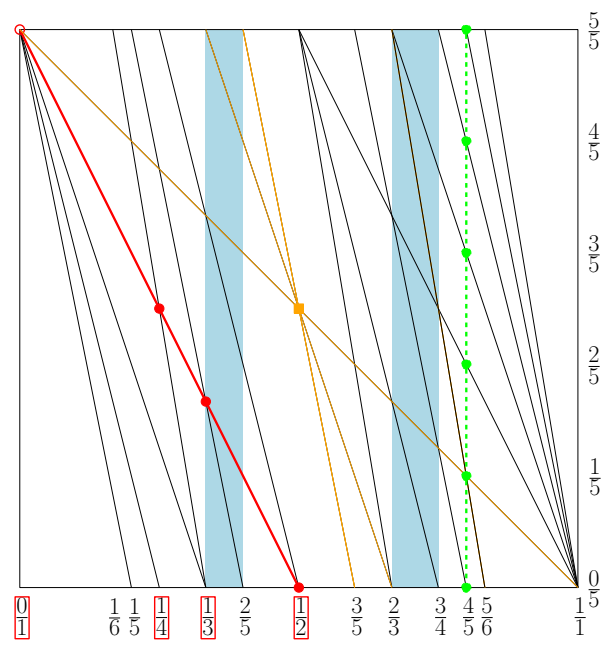

(b)

Fig. 2. (a) Farey Fan of order 6. (b) Illustration of Properties 1 to 4 from Section 3.

Definition 3. Let $S$ be a DSS of length n. Facet $(S)$ is the facet equal to $\mathcal{P}(S)$ in the Farey fan of order $n$.

Moreover, from Proposition 1, a one-to-one correspondence can be defined between a facet and the characteristic point of the facet.

Definition 4. Let $f$ be a facet of the Farey fan of order $n$. We denote by $C$ Point $(f)$ the point $v$ of $f$ such that, if $v=\left(\frac{p}{q}, \frac{r}{q}\right)$, then $(p, q, r)$ are the minimal characteristics of the DSS Facet ${ }^{-1}\left(\right.$ CPoint $\left.^{-1}(v)\right)$.

The Farey Fan of order 6 is depicted in Figure 2(a). The characteristic points of a few facets are depicted. Note that three types of facets can be identified:

- quadrilateral facets (in orange in Figure 2(a));

- upper triangular facets (in green in Figure 2(a));

- lower triangular facets (in blue in Figure 2(a)).

Consider now the following problem:

Problem 1. Given a DSL $L$ of characteristics $\left(a^{\prime}, b^{\prime}, \mu^{\prime}\right)$ and two points $P\left(x_{P}, y_{P}\right)$ and $Q\left(x_{Q}, y_{Q}\right)$ of this DSL, compute the minimal characteristics $(a, b, \mu)$ of the DSS $S=\left\{(x, y) \in L \mid x_{P} \leq x \leq x_{Q}\right\}$.

After a translation of the characteristics of $L$ such that $P$ is set to the origin $\left(\mu \leftarrow \mu+a x_{P}-b y_{P}\right)$, this problem is equivalent to the following one:

Problem 2. Given a point $\boldsymbol{\Lambda}\left(\frac{a}{b}, \frac{\mu}{b}\right)$ and a point $Q\left(x_{Q}, y_{Q}\right)$, find the point $v$ of the Farey fan of order $n=x_{Q}$ such that $\boldsymbol{\Lambda} \in \operatorname{CPoint}^{-1}(v)$.

In other words, the problem is to find the characteristic point of the facet of $\mathcal{F}_{n}$ containing $\boldsymbol{\Lambda}$. 
All in all solving Problem 2 is equivalent to performing a point location in an arrangement of lines. However, the number of facets in the Farey fan of order $n$ (which is equal to the number of DSS of length $n$ ) is in $\mathcal{O}\left(n^{3}\right)$ 9102, and point location algorithms in such a structure are expensive in term of both time and space complexity [13. This brute force approach is then less efficient than classical DSS recognition algorithms [517117].

In the following sections, we revisit the approach proposed by 12 and present an algorithm to solve Problem 2 in time complexity $\mathcal{O}(\log n)$, without explicitly computing the Farey fan. In the next section, we recall several structural and arithmetical properties of the Farey fan, and derive some very useful corollaries. These properties are the core of the algorithm detailed in section 4 .

\section{Properties of the Farey Fan}

The Farey series of order $n$ is the set of irreducible fractions in $[0,1]$ of denominator lower than or equal to $n$ [8]. All the properties below are illustrated in Figure 2(b) in the Farey fan of order 6. The first three properties are from 12 and the reader is invited to consult this reference for the proofs, that are fairly simple.

Property 1 ([12]). The abscissas of intersections of a ray $R(x, y)$ of $\mathcal{F}_{n}$ with other rays are consecutive terms of a Farey series of order $\max (x, n-x)$.

In Figure 2(b), the abscissas of the intersections between the ray $R(2,1)$, depicted in red, and the other rays of $\mathcal{F}_{6}$ are consecutive terms of the Farey series of order $4=\max (2,6-2)$.

Property 2 ([12]). Let $f_{i}$ and $f_{i+1}$ be two consecutive fractions of the Farey series of order $n$. In the interval $f_{i}<\alpha<f_{i+1}$, there is no intersection of rays. Thus, in this interval the Farey fan is a simple ladder of rungs.

In Figure 2(b), two ladders are depicted in blue for $f_{i}=\frac{1}{3}$ and $f_{i}=\frac{2}{3}$.

Property 3 ([12]). Let $v\left(\frac{p}{q}, \frac{r}{q}\right), 0 \leq p \leq q \leq n$, be a point of $\mathcal{F}_{n}$. Let $R\left(x_{0}, y_{0}\right)$ be the ray of minimum slope passing through $v$. The other rays passing through $v$ have a slope equal to $x_{0}+k q$ with $k \in \mathbb{Z}$ and $x_{0}+k q \leq n$.

In Figure 2(b), three rays go through the point $\left(\frac{1}{2}, \frac{1}{2}\right)$ (in orange). The slopes of these rays are equal to $x_{0}=1,3$ and 5 . From this property, we can derive the following corollary.

Corollary 1. Let $v\left(\frac{p}{q}, \frac{r}{q}\right), 0 \leq p \leq q \leq n$, be a point of $\mathcal{F}_{n}$. Let $R(x, y)$ be a ray passing through $p . \stackrel{R}{R}$ is the ray of smallest slope passing through $v$ if and only if $x-q<0$. It is the ray of greatest slope passing through $v$ if and only if $x+q>n$.

Property 4. Let $\frac{p}{q}$ be a fraction of the Farey series of order $n$. The intersection between the line $\alpha=\frac{p}{q}$ and $\mathcal{F}_{n}$ is exactly the set of points $\left(\frac{p}{q}, \frac{r}{q}\right)$ where $r$ takes all the integer values between 0 and $q$. 
Proof. We study the intersection between $R(x, y)$ defined by the equation $\beta=$ $-\alpha x+y$ and $\alpha=\frac{p}{q}$. We get $\beta=\frac{-p x+q y}{q}$. For $0 \leq y \leq x \leq q \leq n$, the quantity $-p x+q y$ takes all the integral values in the interval $[|0, q|]$, which ends the proof.

In Figure 2(b), the intersection between $\alpha=\frac{4}{5}$ (depicted in green) and $\mathcal{F}_{n}$ is the set of points $\left(\frac{4}{5}, \frac{r}{5}\right)$ with $r \in \mathbb{Z}, 0 \leq r \leq 5$. Using Properties 2 and 4 , we can prove the following result to compute the ray of smallest slope in a given point.

Corollary 2. Let $v\left(\frac{p}{q}, \frac{r}{q}\right), 0 \leq p \leq q \leq n$, be a point of $\mathcal{F}_{n}$. Let $\frac{p^{\prime}}{q^{\prime}}$ be the fraction following $\frac{p}{q}$ in the Farey Series of order $n$. The ray of smallest slope passing through $v$ is defined by the point $v$ and the point of coordinates $v^{\prime}\left(\frac{p^{\prime}}{q^{\prime}}, \frac{\left\lfloor\frac{r q^{\prime}}{q}\right\rfloor}{q^{\prime}}\right)$.

Proof. From Property 2, $\mathcal{F}_{n}$ is a ladder in the interval $\left[\frac{p}{q}, \frac{p^{\prime}}{q^{\prime}}\right]$, which means there is no intersection of rays in this interval. From Property 4] we know that there is at least one ray passing through the point $v$. Again from Property 4 , all the rays passing through $v$ cut the line of equation $\alpha=\frac{p^{\prime}}{q^{\prime}}$ in a point $v^{\prime}\left(\frac{p^{\prime}}{q^{\prime}}, \frac{r^{\prime}}{q^{\prime}}\right), r^{\prime} \in \mathbb{Z}$, $0 \leq r^{\prime} \leq q^{\prime}$. Among all these rays, the ray of smallest slope is the one that passes through the point $v_{\max }\left(\frac{p^{\prime}}{q^{\prime}}, \frac{r_{\max }}{q^{\prime}}\right)$ where $r_{\max }$ is the maximal value of $r^{\prime}$ such that $\frac{r^{\prime}}{q^{\prime}} \leq \frac{r}{q} . r_{\max }$ is given by $\left\lfloor\frac{r q^{\prime}}{q}\right\rfloor$ which ends the proof.

\section{Fast Walk in the Farey Fan}

Following Problem 2, we look for the characteristic point of the facet of $\mathcal{F}(n)$ containing a given point $\boldsymbol{\Lambda}\left(\frac{a}{b}, \frac{r}{b}\right)$. From Proposition 1, Section 2.2 and Property 4 we have the following characterization of the characteristic point.

Property 5. A point $v\left(\frac{p_{v}}{q_{v}}, \frac{r_{v}}{q_{v}}\right)$ is the characteristic point of a facet if and only if:

1. either $v$ is the intersection of the two lower edges:

(a) the ray supporting the right lower edge is the one of smallest slope in $v$;

(b) the ray supporting the left lower edge is the one of greatest slope in $v$;

2. or $v$ is on the unique lower edge and more than one ray passes through the point $\left(\frac{p_{v}}{q_{v}}, \frac{r_{v}+1}{q_{v}}\right)$

As in [12, the algorithm consists of three steps that are detailed in the following sections:

1. Find the ladder to which $\boldsymbol{\Lambda}$ belongs;

2. Locate the highest ray that lies on or below $\boldsymbol{\Lambda}$ : this ray supports a lower edge of the facet (Section 4.2, Algorithm 1);

3. Walk along the $\operatorname{ray}(\mathrm{s})$ to determine the characteristic point (Section 4.3. Algorithm 2).

Particular cases where $\boldsymbol{\Lambda}$ is a point of $\mathcal{F}_{n}$ (either on a ray, or a vertex) are eluded, so that the focus is done on the general case. However, these particular cases are not complicated to handle. 


\subsection{Find the Ladder}

Given a point $\boldsymbol{\Lambda}\left(\frac{a}{b}, \frac{\mu}{b}\right)$, finding the ladder to which $\boldsymbol{\Lambda}$ belongs in $\mathcal{F}_{n}$ is equivalent to finding the two fractions with a denominator smaller than $n$ closest to $\frac{a}{b}$ (greater and lower). We look for two fractions $f=\frac{p}{q}$ and $g=\frac{p^{\prime}}{q^{\prime}}$ such that $q \leq n, q^{\prime} \leq n, f \leq \frac{a}{b} \leq g$, and there is no fraction of denominator smaller or equal to $n$ neither between $f$ and $\frac{a}{b}$ nor between $\frac{a}{b}$ and $g$.

This problem is closely related to the computation of the best rational approximation of a number, for which solutions using the decomposition into continuous fractions exist [8]. However, we do not need only the best approximation, which is either the closest lower or closest greater fraction, but also the other one. To solve this problem, we use the algorithm of Charrier and Buzer [3]. This algorithm aims at computing the approximation of any real number by rational numbers of bounded denominator and straightforwardly solves our problem in $\mathcal{O}(\log (n))$. Moreover the algorithm is simple to implement and does not require continuous fractions computations.

\subsection{Locate a Lower Edge}

At this point, we work in a ladder defined by two fractions $f=\frac{p}{q}$ and $g=\frac{p^{\prime}}{q^{\prime}}$ of $\mathcal{F}_{n}$. This step consists in localising $\boldsymbol{\Lambda}$ in the ladder by computing the highest ray under $\Lambda$ in $\mathcal{F}_{n}$. In [12, this step is performed as a binary search among the rays of the ladder. However, each stage of the binary search requires to solve a diophantine equation with the extended Euclidean algorithm, reaching a total complexity of $\mathcal{O}\left(\log ^{2} n\right)$.

Our algorithm, presented in Algorithm 1 and illustrated in Figure [3, also performs a dichotomy (line 4), but only on the rays of smallest slope passing through the points of abscissa $\frac{p}{q}$ (in red in Figure 3).

Thanks to Property 4 , this set of points can be defined as on line 1, and the rays of smallest slope are computed in time $\mathcal{O}(1)$ in the ladder using Corollary 2 (line 2). On line 4, the ray of greatest slope is computed from the ray of smallest

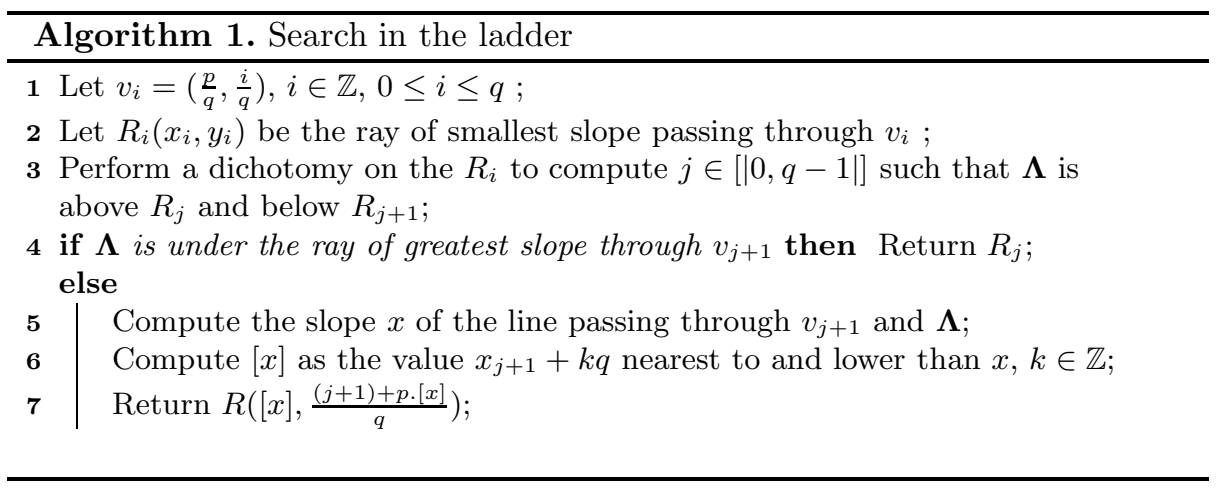


slope thanks to Property 3. On line 5, the value $x$ is not an integer value, but the closest lower ray can be easily computed using Property 3 .

In Figure 3, on the left, the point $\boldsymbol{\Lambda}$ is located under the ray of greatest slope passing through $v_{j+1}$ (in green, line 4 in Algorithm 1), $R_{j}$ is returned. On the right, the point $\Lambda$ is in between the rays passing through $v_{j+1}$ : the slope of the line passing through $v_{j+1}$ and $\boldsymbol{\Lambda}$ is computed (in blue, line 5 in Algorithm 1), and is rounded to find the nearest lower ray.
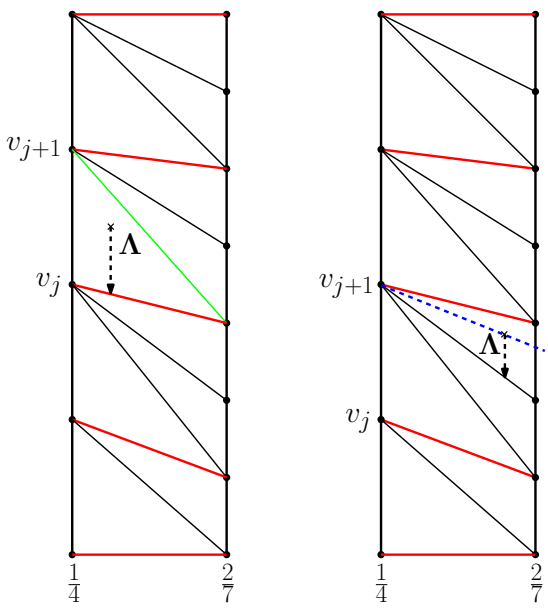

Fig. 3. Illustration of Algorithm 1 the dichotomy is performed on the red rays only

\subsection{Find the Characteristic Point}

Let us denote by $M$ and $N$ the two points defined as the intersection between the ray $R(x, y)$ returned by Algorithm 1 and the vertical lines defining the ladder, i.e. $\alpha=\frac{p}{q}$ and $\alpha^{\prime}=\frac{p^{\prime}}{q^{\prime}}$ as defined in Section 4.1. The segment $[M N]$ is part of a lower edge of the facet of $\mathcal{F}_{n}$ containing $\boldsymbol{\Lambda}$ in $\mathcal{F}_{n}$.

The first step of the algorithm detailed in Algorithm 2 is to compute the extremities of the lower edge containing $[M N]$. To do so, the key point is to use Property 1 to characterize the points of intersection between a ray and other rays. Given a ray $R(x, y)$ of the Farey Fan $\mathcal{F}_{n}$ and a point $v\left(\frac{p}{q}, \frac{r}{q}\right)$ on this ray, $v$ is the crossing point of several rays if and only if $q \leq \max (x, n-x)$. Thus, the abscissa of the left (resp. right) extremity of the lower edge is given by the term of the Farey series of order $\max (x, n-x)$ lower than (resp. greater than) and closest to $\frac{p}{q}$ (resp. $\frac{p^{\prime}}{q^{\prime}}$ ) (line 1 of Algorithm 2). Given a fraction, computing the next term in a Farey series of given order cannot be solved in constant time but requires a call to the extended Euclidean algorithm. From these two fractions $\frac{\underline{p}}{\underline{q}}$ and $\frac{\bar{p}}{\bar{q}}$ we compute the two points $\underline{O}$ of $R$ with abscissa equal to $\frac{p}{\underline{q}}$ and $\bar{O}$ of $\frac{\underline{q}}{R}$ with abscissa equal to $\frac{\bar{p}}{\bar{q}}$ (line 2 ). 
At this point, $[\underline{O} \bar{O}]$ is a lower edge of the facet containing $\boldsymbol{\Lambda}$. Then, the three cases illustrated in Figure 4 can occur: either $\underline{O}$ or $\bar{O}$ is the characteristic point (case (a) and (b)), or not (case (c)). We use Property 5 to distinguish between these cases:

- if $R$ is the ray of smallest slope in $\underline{O}$, then $\underline{O}$ is the characteristic point: the condition line 3 refers to Corollary 1 ,

- if $R$ is the ray of greatest slope in $\bar{O}$, then $\bar{O}$ is the characteristic point: the condition line 4 refers to Corollary 1 ;

- otherwise, the facet is lower triangular, and the abscissa of the characteristic point is given by the mediant of the abscissae of the lower edge extremities, i.e. $\underline{O}$ and $\bar{O}$ (direct consequence of Property 1): on line 5, the mediant is computed, and the point of $R$ with this abscissa is the characteristic point.

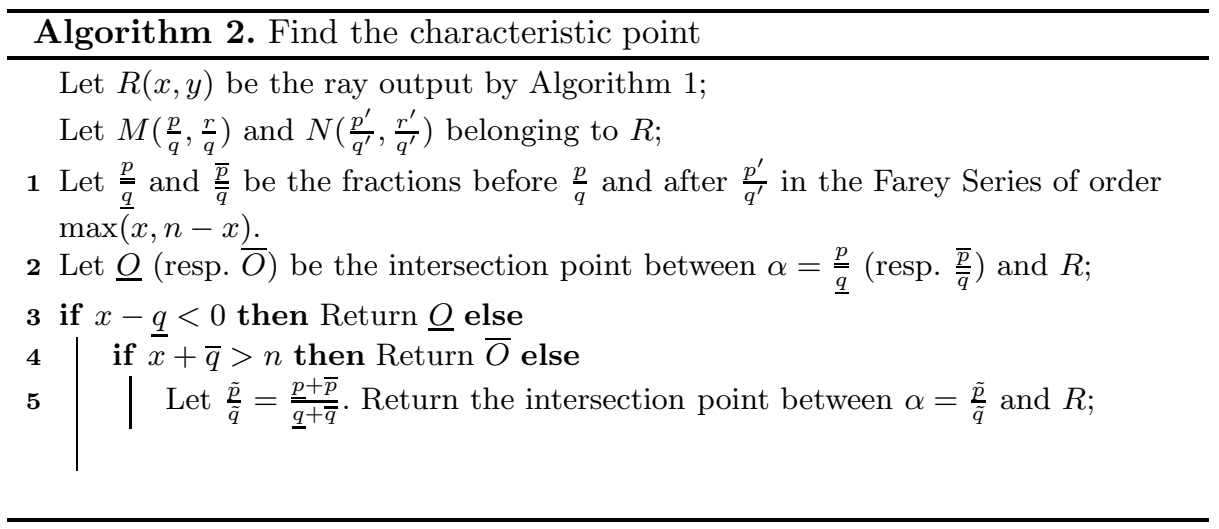

\subsection{Complexity}

Lemma 1. The complexity of the algorithm described in Section 4 is in $\mathcal{O}(\log (n))$, where $n$ is the length of the DSS.

Proof. We assume a computing model where standard arithmetic operations are done in constant time. Finding the ladder is done using the algorithm of Charrier and Buzer [3] that has a complexity of $\mathcal{O}(\log (n))$.

The localization of a lower edge is done with Algorithm 1 the computation of the $R_{i}$ (line 2) is done in constant time thanks to Corollary 2, such that the global complexity of lines 2 and 3 is $\mathcal{O}(\log (q))$ with $q \leq n$. The operations done in lines 4 to 7 are done in constant time, and the complexity of Algorithm 1 is in $\mathcal{O}(\log (q))$.

Algorithm 2 performs the last step of the algorithm. On line 1, two calls to the extended Euclidean algorithm are necessary to compute the lower edge extremities, which takes $\mathcal{O}(\log (n))$. All the other operations of this algorithm take $\mathcal{O}(1)$, which ends the proof. 


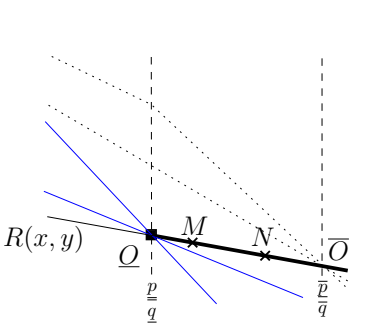

(a)

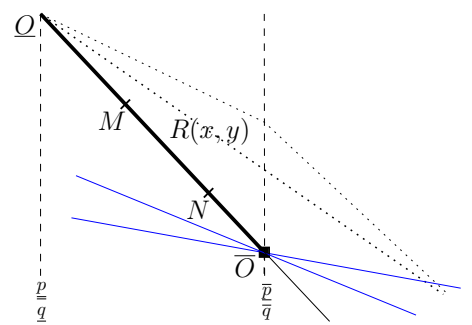

(b)

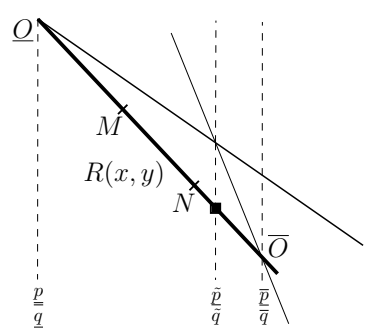

(c)

Fig. 4. Three cases for the lower edge $[\underline{O} \bar{O}]$ : (a) all the rays passing through $\underline{O}$ (in blue) have a slope greater than $x$ and $\underline{O}$ is the characteristic point; (b) all the rays passing through $\bar{O}$ (in blue) have a slope smaller than $x$ and $\bar{O}$ is the characteristic point; (c) neither $\underline{O}$ nor $\bar{O}$ is the solution, and the characteristic point is found with a mediant computation

Algorithm 2 can actually be optimized so that the call to the extended Euclidean algorithm (line 1) is not always necessary. These optimizations consist in the study of particular cases that are not presented here to keep the algorithm as clear as possible. All in all they do not change the theoretical complexity but lower the constant term, and slightly improve the practical efficiency.

This algorithm solves Problem 2 in $\mathcal{O}(\log (n))$ where $n$ is the order of the Farey fan. From the equivalence of Problems 1 and 2, this algorithm also solves Problem 1 in logarithmic time where $n$ is the length of the DSS.

\section{Experimentation}

We have implemented the presented algorithm using the open-source library DGtal [1]. The algorithm is very easy to implement and does not require continuous fractions implementation as in 1415]. The algorithms of Said and Lachaud 1415. being implemented in this library, comparing the algorithms was then an easy task. We also conducted the experimentation along the same protocol as the one they proposed as a test file in DGtal. Basically, the idea is to randomly choose a maximal value $N$ for the parameter $b$ of the DSL ( $a$ is smaller than $b$ ), then fix a maximal value for the length $n$ of the DSS, and finally randomly choose a shift $\mu$ and the abscissa of the DSS first point. Each experiment is conducted for 10000 randomly chosen parameters.

The algorithms are executed to compute the characteristics of the DSS contained in the DSL. For each algorithm, the total running time is measured and divided by the total number of trials.

Figure 5 represents the results obtained for $N=10^{6}$ in (a), $N=10^{9}$ in (b) and $n$ taking all the value of the form $10.2^{k}$ in the interval $[10, N]$. The graph represents the execution time in ms versus the maximal length $n$ of the DSS.

\footnotetext{
1 The $\mathrm{C}++$ code of this algorithm is freely available on the webpage http://www.gipsa-lab.grenoble-inp.fr/ isabelle.sivignon
} 
The first observation is that SmartDSS is clearly slower than the other two algorithms. The second observation concerns the behaviour of the curves: the execution time increases with $n$ for our algorithm while it decreases for ReversedSmartDSS. This is consistent with the complexities of the algorithms. The complexity of our algorithm is logarithmic in the length of the DSS while the complexity of ReversedSmartDSS depends on the difference of depth of the slope of the DSL and the slope of the DSS. Consequently, our algorithm is more efficient for short DSSs, while for ReversedSmartDSS, the greater is $n$, the smaller is the difference of slopes, and the more efficient is the algorithm.

It is thus interesting to study the value of $n$ for which the two curves cross each other. We see in Figure 5 that this value is $10^{4}$ for $N=10^{6}$ and $10^{6}$ for $N=10^{9}$. Other experiments show that this value is $10^{3}$ for $N=10^{4}$ and that the threshold ratio $\frac{n}{N}$ below which our algorithm is faster tends to decrease with $N$.

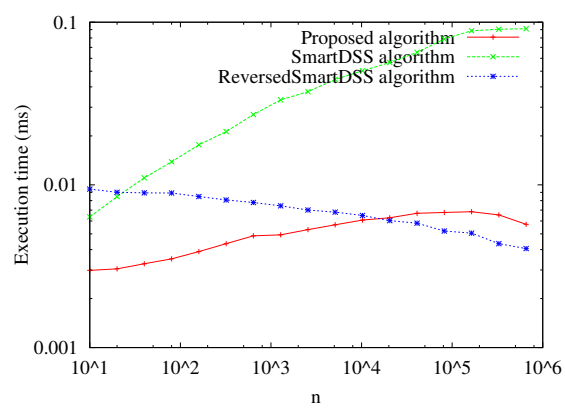

(a) $N=10^{6}$

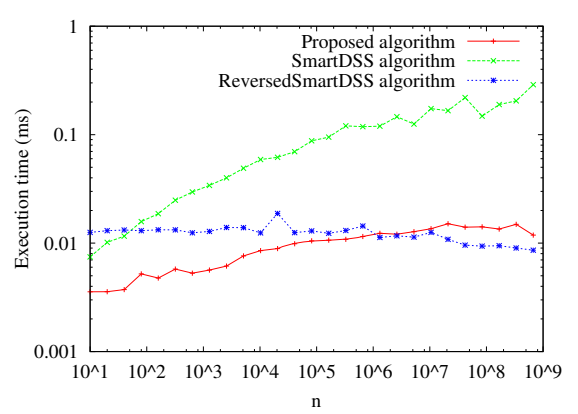

(b) $N=10^{9}$

Fig. 5. Runtime comparison of our algorithm and the algorithms of [14]15]

\section{Conclusion}

We have proposed an algorithm to compute the characteristics of a DSS which is a subsegment of a DSL of known characteristics. We use the Farey fan and its numerous arithmetical properties to design a very efficient both theoretically and practically, and easy to implement algorithm to solve this problem.

We prensented the algorithm in the case where the DSL parameters are rational fractions. However, it can be straightforwardly extended to deal with irrational parameters.

The exprimental section has shown that our algorithm is faster than the ReversedSmartDSS algorithm when the length of the DSS is sufficiently smaller than the DSL period. This suggests that the ReversedSmartDSS algorithm should be prefered when the DSL parameters are issued from the recognition of a DSS on an image, and that our algorithm would perform better to draw a DSS given by floating-point vertices on an image. It would however be interesting to deepen this comparison. 


\section{References}

1. DGtal: Digital Geometry Tools and Algorithms Library, http://libdgtal.org

2. Berenstein, C., Lavine, D.: On the number of digital straight line segments. IEEE Trans. on Pattern Anal. and Mach. Intell. 10(6), 880-887 (1988)

3. Charrier, E., Buzer, L.: Approximating a real number by a rational number with a limited denominator: A geometric approach. Discrete Applied Mathematics 157(16), 3473-3484 (2009)

4. Coeurjolly, D., Sivignon, I., Dupont, F., Feschet, F., Chassery, J.-M.: On digital plane preimage structure. Discrete Applied Mathematics 151(1-3), 78-92 (2005)

5. Debled-Rennesson, I., Reveillès, J.-P.: A linear algorithm for segmentation of digital curves. Inter. Jour. of Pattern Recog. and Art. Intell. 9(6), 635-662 (1995)

6. Dorst, L., Smeulders, A.N.M.: Discrete representation of straight lines. IEEE Trans. on Pattern Anal. and Mach. Intell. 6(4), 450-463 (1984)

7. Dorst, L., Smeulders, A.W.: Discrete straight line segments: Parameters, primitives and properties. In: Vision Geometry. Contemporary Mathematics, pp. 45-62. Am. Math. Soc. (1991)

8. Hardy, G.H., Wright, E.M.: An Introduction to the Theory of Numbers. Oxford Society (1989)

9. Klette, R., Rosenfeld, A.: Digital geometry - geometric methods for digital picture analysis. Morgan Kaufmann (2004)

10. Koplowitz, J., Lindenbaum, M., Bruckstein, A.: The number of digital straight lines on an $n \times n$ grid. IEEE Trans. on Info. Theory 36(1), 192-197 (1990)

11. Kovalevsky, V.: New definition and fast recognition of digital straight segments and arcs. In: Inter. Conf. on Patt. Anal. and Mach. Intell., vol. 2, pp. 31-34 (1990)

12. McIlroy, M.D.: A Note on Discrete Representation of Lines. AT\&T Technical Journal 64(2), 481-490 (1985)

13. Wein, R., Fogel, E., Zukerman, B., Halperin, D.: CGAL - 2D Arrangements, http://www.cgal.org/Manual/3.3/doc_html/cgal_manual/Arrangement_2/ Chapter_main.html

14. Said, M., Lachaud, J.-O., Feschet, F.: Multiscale Discrete Geometry. In: Brlek, S., Reutenauer, C., Provençal, X. (eds.) DGCI 2009. LNCS, vol. 5810, pp. 118-131. Springer, Heidelberg (2009)

15. Said, M., Lachaud, J.-O.: Computing the Characteristics of a SubSegment of a Digital Straight Line in Logarithmic Time. In: Debled-Rennesson, I., Domenjoud, E., Kerautret, B., Even, P. (eds.) DGCI 2011. LNCS, vol. 6607, pp. 320-332. Springer, Heidelberg (2011)

16. Sivignon, I., Dupont, F., Chassery, J.M.: Digital intersections: minimal carrier, connectiviy and periodicity properties. Graphical Models 66(4), 226-244 (2004)

17. Troesch, A.: Interprétation géométrique de l'algorithme d'euclide et reconnaissance de segments. Theor. Comput. Sci. 115(2), 291-319 (1993) 